\title{
Mechanistic Understanding of Surface Migration Dynamics with DNA Walkers \\ Yancheng Du${ }^{1}$, Jing Pan², Hengming Qiư ${ }^{1}$, Chengde $\mathrm{Mao}^{3}$, and Jong Hyun Choi ${ }^{1}$, * \\ ${ }^{1}$ School of Mechanical Engineering, Purdue University \\ ${ }^{2}$ Department of Mechanical and Aerospace Engineering, University of Florida \\ ${ }^{3}$ Department of Chemistry, Purdue University \\ ${ }^{*}$ Corresponding author: jchoi@purdue.edu
}

\begin{abstract}
Dynamic DNA walkers can move cargoes on a surface through various mechanisms including enzymatic reactions and strand displacement. While they have demonstrated high processivity and speed, their motion dynamics are not well understood. Here, we utilize an enzyme-powered DNA walker as a model system and adopt a random walk model to provide new insight on migration dynamics. Four distinct migration modes (ballistic, Lévy, self-avoiding, and diffusive motions) are identified. Each mode shows unique step time and velocity distributions which are related to mean squared displacement (MSD) scaling. Experimental results are in excellent agreement with the theoretical predictions. With a better understanding of the dynamics, we performed a mechanistic study, elucidating the effects of cargo types and sizes, walker sequence designs, and environmental conditions. Finally, this study provides a set of design principles for tuning the behaviors of DNA walkers. The DNA walkers from this work could serve as a versatile platform for mathematical studies and open new opportunities for bioengineering.
\end{abstract}

Keywords: DNA nanotechnology; DNA walker; random walk; surface migration; migration dynamics 


\section{INTRODUCTION}

As a rapidly growing field, DNA nanotechnology uses nucleic acids as engineering materials. ${ }^{1-}$

4 The advantages of using DNA as structural components include excellent sequence complementarity and structural predictability. These properties allow for construction of complex self-assembled structures such as two-dimensional (2D) lattices ${ }^{5,6}$ and 3D polyhedrons..$^{7-9}$ In parallel to static structures, dynamic nanodevices with a new level of programmability were also developed, including switches, ${ }^{10,11}$ circuits $^{12-15}$ and reconfigurable structures. ${ }^{16,17}$ In particular, DNA walkers were inspired by intracellular protein motors and first demonstrated with hybridization and strand displacement. ${ }^{18,19}$ By sequentially adding DNA strands, the walkers move along a predesigned track and make steps in series through basepairing, dissociation, and branch migration. For autonomous directional motions, multiple mechanisms ${ }^{20}$ have been developed, including strand displacement, ${ }^{21-23}$ enzymatic reaction ${ }^{24-}$ ${ }^{26}$ and light-induced reconfiguration. ${ }^{27}$ DNA walkers may also be programmed to execute complex tasks such as molecular assembly, ${ }^{28}$ cargo sorting, ${ }^{29}$ drug release ${ }^{30}$ and organic synthesis. ${ }^{31,32}$

Over the past decade, significant efforts have been made to achieve faster and more processive DNA motors. Current walkers can move tens of microns over 10 hours with an average velocity of several nanometers per second. ${ }^{33-36}$ Migrations of these motors on surfaces involve multiple mechanisms and design parameters. ${ }^{37-40}$ Understanding the kinetics of translocation processes is essential to improve the performances of movements. Based on the analysis of single turnovers (i.e., walking cycle), it has been shown that environmental conditions affect the kinetics of enzyme cleavage and associations/dissociations between strands. ${ }^{23,}{ }^{41}$ Theoretical random motion models such as self-avoiding walk were used to understand migration mechanisms. ${ }^{42,}{ }^{43}$ Mechanical models on DNA binding revealed the effects of tensions between walker feet and tracks ${ }^{44}$ and force dependence of motors. ${ }^{45}$ In terms of thermodynamics, free energy barriers and efficiencies of different DNA motors were compared. ${ }^{46}$ These studies provided design guidelines for building walkers with better motility and processivity, while focusing more on a single mode of migration. In actual experiments, however, DNA walkers have been observed to move with more complexity and the same design guidelines may not be applicable for different types of motions. For example, variations in velocity distribution and some type of hopping have been reported previously, ${ }^{47}$ and the relevant mean squared displacement (MSD) scaling is larger than theoretical models. ${ }^{48}$ Therefore, a more complete, universal model for different motions is needed for understanding the moving dynamics.

In this work, we introduce a random walk model based on the continuous time random walk $(\mathrm{CTRW})^{49}, 50$ to elucidate the fundamental relationship between MSD scaling of different motions and distributions of steps and velocities. We use an enzyme powered DNA walker, which migrates on surfaces decorated with RNA fuels, as the model system. Synthetic vesicles (SV) and polystyrene (PS) particles were modified with DNA strands and driven by an enzymatic cleavage of RNA fuels via a burnt-bridge mechanism. Particle movements were imaged with total internal reflection fluorescence (TIRF) microscopy. Each trajectory is segmented into a series of steps which are identified as four distinct walking modes - ballistic, 
Lévy, self-avoiding and diffusive. With improved understanding of the dynamics, we investigated the effects of design parameters in the walker system including types and sizes of cargoes, strand designs, and environmental conditions. A set of design principles was extracted from the results. We find that a particular migration mode can be promoted or suppressed with changes in cargo sizes or strand designs. DNA motility (measured by velocities) and processivity (measured by step lengths) are influenced by walker lengths and environmental conditions. Thus, DNA walkers could serve as experimental platforms for mathematical models and their performances may be tuned to suit different applications.

\section{Results}

\section{Burnt-bridge mechanism}

A burnt-bridge mechanism was first proposed in 2005 and has been widely used in walker designs. ${ }^{51}$ Several enzymes were used to realize the mechanism including DNAzymes, ${ }^{25,52}$ nicking restriction enzymes, ${ }^{51}$ and exonuclease $1 \mathrm{II} .{ }^{53}$ In this work, we used an RNAse $\mathrm{H}$ enzyme due to its high processivity. 47,48 The enzyme recognizes and cleaves the RNA strand in a DNA/RNA hybrid, thus the consumed RNA strands cannot be used again as fuels. A single turnover (i.e., a single walking cycle) may be considered as a four-step process illustrated in Figure 1a. (i) An enzyme (green) recognizes and binds to the DNA/RNA hybrid. (ii) The enzyme digests the RNA fuel (blue) and releases the DNA walker strand (black). (iii) The released DNA finds the next available RNA fuel. (iv) The walker strand moves to the new RNA fuel via branch migration and carries the cargo particle (orange) along. Driving force in the mechanism is created by the bias of consumed fuels. These fuels are permanently disabled, which makes the moving particles preferentially explore a region of unvisited sites rather than an adjacent region of previously visited sites. Thus, the particle behaviors deviate from the Brownian motion.

MSD is commonly used to analyze the characteristics of movements. ${ }^{54}$ MSD measures the time dependence of particle migration in space as

$$
M S D=<\left(x-x_{0}\right)^{2}>
$$

where $x$ represents the position of the particle in space. MSD scales as

$$
M S D \sim t^{\alpha}
$$

where the scaling exponent $\alpha$ varies with different types of motions. In theory, Brownian diffusion follows $M S D \sim t$ with $\alpha=1$, while the MSD of self-avoiding walk has been shown as $<x^{2}>\sim t \cdot \log (t)$, with $\alpha \approx 1.4$ in large timescale. ${ }^{55-57}$ For ballistic motion, the MSD scaling is $\alpha=2$.

\section{Classification of walking modes}

We performed walking experiments with DNA decorated particles and characterized their motions with MSD analysis ${ }^{47}$. The particles were placed on a $2 \mathrm{D}$ surface modified with high density RNA fuels, and enzymes were added to drive random movements. Fluorescent probes were attached to the particles for imaging and the trajectories were recorded with TIRF microscopy. The recorded data were fitted to a Gaussian point spread function to obtain superresolution localizations with a time interval of 5 seconds. We then calculated the MSD scaling of trajectories and classified the particles based on the scaling. A typical trajectory of a particle 
is shown in Figure $1 \mathrm{~b}$ and Movie 1. The scaling of the trajectory analyzed as a whole has $\alpha=$ 1.26, which is close to self-avoiding motion. However, we also observed that the walker demonstrates different patterns along its trajectory. At the beginning, the particle showed a tendency moving away from the starting spot (shown in green) and searched around consistently (orange). Then, the particle turned into a migration mode with large velocity with irregular hops (red). The particle also demonstrated movements restricted in a small region (gray). Later the particle escaped from the region and moved with steady paces (blue) to the end of the measurement.

Based on the observation, statistical analysis of a single trajectory does not provide enough physical insight. Segmentation of trajectories is needed to understand the dynamics. Following criteria are set to divide between different segments (i.e. steps). (1) If the displacement of a particle is smaller than $20 \mathrm{~nm}$ for a consecutive time (e.g., >10 seconds), it is classified as a pause. Pause indicates that the particle shows no significant movement (within the measurement error). Before and after the pause, we regard the trajectory as two different steps. (2) If a particle has a displacement larger than $300 \mathrm{~nm}$ between localizations, we classify it as a leap. A large leap distorts the MSD scaling; thus, we count them as different steps before and after the leap. (3) Directional changes are commonly used to differentiate steps $^{58,59}$. If the moving direction of a particle turns larger than 10 degrees from the original direction, it is classified as a turn and divided into two steps. Steps are analyzed individually and classified into different modes based on $\alpha$ value.

These criteria were applied to segment the particle trajectory in Figure 1b into steps. Each step was analyzed and classified. Displacement of the particle in each step is recorded as step length and the time span of each step is registered as step time. Different step lengths and step times will affect the MSD scaling of the particle. Figure 1c shows an MSD plot of four representative trajectories in distinct migration modes. A color scheme is used for distinguishing different migration modes throughout the paper. With $\alpha \geq 2.0$, the step is classified as ballistic motion (shown in red). Movements with heavy tail distributed step lengths and $1.6 \leq \alpha<2.0$ are classified as Lévy flights (blue). ${ }^{58}$ Their MSD demonstrates a larger scaling than the theoretical prediction of self-avoiding motion (green). Experimental selfavoiding migration is defined as $1.1 \leq \alpha<1.6$ (shown in orange). The range $0.8 \leq \alpha<1.1$ is used to classify diffusions and the plot in gray color is close to the theoretical diffusion limit with $\alpha=1$. For $\alpha<0.8$, the particles do not show significant movement, thus their motion is not included in the analysis. All the MSD plots in a short time period follow the $\alpha=1$ line and differentiate into four migration modes at a larger timescale. The transition results from the time-averaged MSD used in our analysis, which shows MSD close to diffusion at the beginning and provides a more accurate estimation of overall MSD in the long term ${ }^{60}$.

\section{Comparison between different migration modes}

To better understand the differences between four modes of migration, we performed experiments with different types of particles under various environmental conditions with distinct sequence designs (see SI for experimental details). In addition, part of vesicle migration data were retrieved from our previous study ${ }^{47}$ and analyzed with the segmentation 
method. The particle motions were classified and analyzed to compare their characteristics. Exemplary trajectories of single particles in distinct modes are shown in Figure 2a (also see Movies 2-5). A total of 747 trajectories are analyzed and the distributions of step time and velocity are shown as histograms in Figure $\mathbf{2 b - 2 c}$. The histograms are fitted with Gaussian functions to extract the motion characteristics. It is noticeable that the distributions of step time become narrower from ballistic to diffusive modes. Velocity distributions show distinctions in the high velocity range $(>8 \mathrm{~m} / \mathrm{s}$ ). For ballistic motion (red), the particle migrates similar to super-diffusion while occasionally performing long distance jumps. These jumps shift the MSD from a super-diffusive range to the ballistic domain due to drastic displacements occurred. The velocity distribution of ballistic motions demonstrates that while the particles have velocities mostly in a small velocity range $(0 \sim 8 \mathrm{~nm} / \mathrm{s})$, they show much higher probability of high speeds (>8 nm/s) compared to other motions. Lévy (blue) and self-avoiding (orange) walks are similar in shapes of trajectories. However, the trajectory of a self-avoiding walk shows more turns and forms more clusters of localizations, while a Lévy motion is more persistent in moving direction and has less localization clusters. Differences also show that Lévy walkers have relatively broader distributions. Especially for the velocity distribution in the range $>8 \mathrm{~nm} / \mathrm{s}$, Lévy particles have large velocities and do not fit with the constant velocity assumption in the selfavoiding model. The randomness in velocities gives a larger scaling to Lévy flight compared to typical $\alpha$ value of $\sim 1.4$ in self-avoiding motion. The trajectory of diffusion (gray) shows frequent changes in moving directions and the trajectory coils around in a relatively small area. These particles are even narrower in step time and velocity distributions. Their frequent changes in the direction result in small step lengths and narrow spatial domains explored. Further, diffusive trajectories may intersect their own tracks and become trapped, which significantly reduces their velocity and MSD scaling.

\section{Random walk model with random velocities}

To better understand the physical origin of different migration patterns, we have adopted a random walk model with random velocities, proposed by Zaburdaev et $\mathrm{al}^{61}$. Step time and velocity distributions are introduced to better represent inconsistent velocities in experiments ${ }^{62}$ and elucidate the scaling of different migration modes. In our analysis, the distributions of velocity $(v)$ and step time $(\tau)$ are modelled as power law functions with heavy tails in general forms of $h(v) \propto|v|^{-1-2 \delta}$ and $F(\tau) \propto \tau^{-1-\gamma}$, respectively. Here, $\delta$ and $\gamma$ are parameters measuring the broadness of their respective distributions. The probability for particles to change the migration velocity $\varphi(x, t)$ is governed by

$$
\varphi(x, t)=\int_{-\infty}^{+\infty} d v \int_{0}^{t} \varphi\left(x-v \tau^{*}, t-\tau^{*}\right) h(v) \mu\left(\tau^{*}\right) d \tau^{*}+n_{0}(x) \delta(t) .
$$

where $n_{0}(x)$ is the initial spatial distribution of particles. $\mu\left(\tau^{*}\right)$ is the distribution of time during which velocity does not change. Thus, particles at time $t-\tau^{*}$ and position $x-v \tau^{*}$ will move with velocity $v$ for time $\tau^{*}$ and arrive at $(x, t)$. The step time distribution is related to $\mu\left(\tau^{*}\right)$ as $F(\tau)=1-\int_{0}^{\tau} \mu\left(\tau^{*}\right) d \tau^{*}$. We integrate all possible events that result in the particles present at position $x$ at time $t$. The number density of particles $n(x, t)$ can then be expressed as 


$$
n(x, t)=\int_{-\infty}^{+\infty} d v \int_{0}^{t} \varphi(x-v \tau, t-\tau) h(v) F(\tau) d \tau
$$

The particle density at a given point $(x, t)$ is a result of particles moving from $(x-v \tau, t-\tau)$ at velocity $v$ and remain with the same velocity for time $\tau$. Therefore, the particle dynamics can be described by velocity and step time distributions.

The scaling is developed similar to the CTRW model (see Experimental and Computational Methods) and shown in Figure 3. Red background represents MSD scaling $\alpha \geq 2.0$ for ballistic motion. Light brown color denotes $1.0<\alpha<2.0$ for super-diffusive movements (Lévy and self-avoiding walks), while diffusive motion has gray background. The velocity distribution affects the scaling mostly by influencing the step lengths of the motion. With a smaller value of $\delta$, the particles will have a broader velocity distribution, implying that long hops and long steps occur more frequently and lead to more ballistic and Lévy motions with less diffusion. As shown in the figure, the gray squares are mostly above $\delta=2$, while red dots will appear more likely with $\delta \leq 2$. The distribution of step times indicates the consistency of the motion. With a broad distribution of step time (i.e., small $\gamma$ value), a particle may move towards a certain direction without pauses or directional changes for a long time, thus it will have large $\alpha$. For instance, when a particle moves along a single direction without a pause, it has an MSD scaling of $\alpha=2$. In contrast, directional changes and pauses will happen more frequently with particles having a narrow step time distribution (i.e., large $\gamma$ value), leading to Brownian diffusion with $\alpha=1$.

The universality of the model is verified by our experiments. The experimental data include over 25 datasets with different cargoes and experimental conditions such as sequence designs and buffer conditions. The trajectories obtained from the experiments were segmented to steps as previously described. Velocities were calculated using a 60-second time interval between localizations. Distributions of velocities and step times were fitted to the power law functions to retrieve $\delta$ and $\gamma$ values. ${ }^{63}$ The results are plotted in Figure 3, where experimental data are shown as squares in the color scheme consistent with the MSD scaling classifications. It is evident that experiment data match with the regimes predicted by the model. Red squares fall in the ballistic region, while Lévy and self-avoiding squares are in the super-diffusion regime. The gray squares are mostly in the diffusive range with several data points being near the borderline. We thus conclude that the model successfully relates statistical distributions to different migration modes regardless of experimental conditions.

\section{Mechanistic studies of surface migration with DNA walkers}

Our improved understanding of the motion dynamics inspired us to investigate how different parameters influence the walking steps and further affect the migration modes. We performed a mechanistic study, exploring two types (SV and PS) of particles. Two different particle sizes (20 and $200 \mathrm{~nm}$ in diameter) were used to compare the effect of walker-fuel valence. The effects of walker length and foothold were also investigated using different sequence designs. Finally, $\mathrm{Mg}^{2+}$ and enzyme concentrations were varied to tune the migration conditions. The motor performances were compared and analyzed in several aspects. For example, certain 
motions were promoted by tuning the cargo size and foothold design. Step lengths were evaluated for the walker processivity. Velocities change with different parameters as kinetics of enzyme cleavage and strand association/dissociation were affected. The mechanistic study is discussed below.

\section{Effects of particles}

The SV and PS particles serve as cargoes carried by DNA walkers. Engineered lipid vesicles are widely used as bioreactors, drug delivery carriers, and protocell models ${ }^{64}$. We synthesized vesicles decorated with DNA walker strands, while PS particles were purchased and modified with DNA strands via click chemistry (see Experimental and Computational Methods). We first compared the performances of the DNA motors carrying different types of particles with similar sizes ( $200 \mathrm{~nm}$ in diameter). The experiments were conducted using 13-nt walker strands with $3 \mathrm{mM} \mathrm{Mg}^{2+}$ and $1 \mathrm{nM}$ enzymes for 1 hour. Figure 4a shows the two pie charts with similar percentages of migration modes. About half of the particles are classified as diffusion. Three quarters of the other half of particles show self-avoiding behaviors, while the rest is Lévy flight. Only few ballistic walkers are identified. No significant differences are observed in the average step lengths of these particles as shown in Figure $\mathbf{4 b}$. The SV and PS demonstrate similar average velocities in a given mode, while distinct velocities were observed in different modes as expected (Figure 4c). For example, their average velocities are approximately $4 \mathrm{~nm} / \mathrm{s}$ in the Lévy mode and $\sim 1 \mathrm{~nm} / \mathrm{s}$ with diffusion. Their average step times also show no significant differences (Figure S1). Thus, we conclude that the nanoparticle cargoes do not affect the motor function of DNA significantly, even with different chemistries.

While particle types show no significant influence, particle sizes can impact in the walker dynamics. A smaller particle has fewer DNA strands on its surface, binding to RNA fuels, which will accelerate the cleavage process and lead to faster kinetics. We compared the performances of PS particles with diameters of 200 and $20 \mathrm{~nm}$. Percentages of different modes are shown in Figure 4d. The pie charts show similar percentages with both sizes. However, step lengths (Figure 4e) and step times (Figure S2) of $20 \mathrm{~nm}$ particles are generally larger than those of $200 \mathrm{~nm}$ particles. The smaller particles can move about 1 micron in each step, while $200 \mathrm{~nm}$ particles have steps smaller than $600 \mathrm{~nm}$. In addition, the average velocities of $20 \mathrm{~nm}$ particles are also significantly faster than those of the larger particles in all modes (Figure 4f).

\section{Effects of walker strands}

The particle motions are driven and controlled by the interactions between walker and fuel strands. Here we introduce different sequence designs to investigate the effects. The PS particles with a diameter of $200 \mathrm{~nm}$ were used for the experiments under concentrations of 3 $\mathrm{mM} \mathrm{Mg}^{2+}$ and $1 \mathrm{nM}$ enzyme. Figure 5 compares free and anchored strands. Free strands are designed such that after cleavage the DNA walkers may be fully released from RNA fuel strands on the surface. In the anchored design, the fuel strands have a 7-nt domain of DNA (nondigestible by the enzyme; shown in black) such that the walker DNA will rely on branch migration for moving towards new fuels. In general, the anchored strands require larger energy fluctuation to overcome hydrogen bonds and result in more diffusive type migration. Figure 
5b shows that about $70 \%$ of the particles with the anchored strands are identified as diffusion, which is significantly higher than those with the free strands $(\sim 50 \%)$. The percentages of selfavoiding and Lévy migrations also decrease compared to those with the free strands. It is noticeable that the anchored walkers have larger step lengths (Figure 5c) and step times (Figure S3) especially in super-diffusive motions. Their steps can be longer than 1 micron, while the steps with free strands are around $600 \mathrm{~nm}$. This may happen because with their shortened search range after enzyme cleavage, they are more consistent in walking directions. As shown in Figure 5d, the free and anchored designs have similar average velocities except for the Lévy case.

The effects of strand lengths are also investigated. We used vesicles with various lengths of walkers and corresponding free-strand fuels $\left(8,13,18\right.$, and 23-nt) with $12 \mathrm{mM} \mathrm{Mg}^{2+}$ and $1 \mathrm{nM}$ enzymes. Figure 6a presents that the percentages of self-avoiding and Lévy motions combined decrease from $\sim 70 \%$ to $\sim 35 \%$ as the strand length increases. On the contrary, the diffusion cases increase significantly with longer strands. Accordingly, high speeds are observed with shorter strands, while longer strands demonstrate slow migration (Figure 6b). For example, 8-nt yields the average velocities of $>1 \mathrm{~nm} / \mathrm{s}$ in all modes, while 23-nt demonstrates only $0.5 \sim 0.9 \mathrm{~nm} / \mathrm{s}$. In terms of step length, two competing mechanisms may play a role. A long strand (e.g., 23-nt) will have better stability, thus it will be less likely to drop off or make long-distance jumps. If the length is too long, however, the particles move slow and have more pauses, resulting in short step lengths. Therefore, 13 or 18-nt walkers mostly lead to long step lengths in all modes, as seen in Figure 6c. Similar patterns can also be observed in average step times (Figure S4).

\section{Effects of environmental conditions}

Environmental conditions may influence DNA/RNA binding and enzyme activity, thereby impacting in migration performances. We examined the effects of various enzyme and $\mathrm{Mg}$ concentrations using SV particles with 18-nt free strands (Figure 7). Figure 7a shows the migration patterns with the enzyme concentration varying from 0.2 to $5 \mathrm{nM}$. While the changes in the percentages of other modes are relatively insignificant, more ballistic and Lévy flights are noticeable with $5 \mathrm{nM}$ enzymes. This is expected as a large amount of enzymes can facilitate cleavage of fuels and may even cause more jumps. This can also be seen in Figure 7b, where average velocities are drastically faster at $5 \mathrm{nM}$ enzymes; they are faster by two folds than those at lower concentrations ( 0.2 and $1 \mathrm{nM})$. High enzyme concentrations also result in shorter step times (see Figure S5) due to more directional changes caused by faster cleavage of fuel strands. However, the step lengths are in the range of $400 \sim 700 \mathrm{~nm}$ and do not vary significantly with enzyme concentration in each mode (Figure 7c).

The effects of $\mathrm{Mg}^{2+}$ concentration was studied at a fixed concentration of $1 \mathrm{nM}$ enzyme. Figure 7d shows that the mode percentages under different $\mathrm{Mg}^{2+}$ concentrations are roughly the same with half of the particles in the self-avoiding regime. We expected that a higher $\mathrm{Mg}^{2+}$ concentration may enhance the walker processivity with stronger binding, yet it may also suppress the enzyme activity due to a reduced cleavage rate. This is evident in Figure 7e. The average velocities show a consistency drop from low to high Mg concentrations in all 
modes. Neither high nor low $\mathrm{Mg}^{2+}$ concentration results in long step lengths (Figure 7f) or step times (Figure S6). At a low $\mathrm{Mg}^{2+}$ concentration, DNA walkers are not stable, while enzyme activity is low at high $\mathrm{Mg}^{2+}$. In general, the particles with $12 \mathrm{mM} \mathrm{Mg}^{2+}$ move in longer steps; the longest step is observed to be $\sim 900 \mathrm{~nm}$ and other conditions do not exceed $500 \mathrm{~nm}$.

\section{DISCUSSION}

In this work, we identified different motions of DNA walkers and used a random walk model to elucidate the physical mechanisms of distinct migration modes. DNA decorated SV and PS particles were exploited as an experimental platform to develop and validate the model. Relationships between the steps and migration modes were revealed, which inspired our mechanistic study to develop guiding principles for tuning walker performances. We showed that different design parameters influence the steps and velocities of particles, together determining the motion dynamics. The findings are summarized as follows.

Effects of surfaces. This study used glass coverslips functionalized with RNA fuel strands. We did not investigate the surface effects, and it is nontrivial to explore various surfaces as an experimental parameter. Different substrates such as gold $(\mathrm{Au})$ films $^{48}$ or glass coverslips ${ }^{47}$ require different chemistry, which may result in distinct chemical environments affecting walker dynamics. These surfaces typically can reach an interval of 10 to $20 \mathrm{~nm}$ between fuel strands. DNA origami may improve the inter-fuel spacing to several nanometers and allow for intricate arrangements of fuels to achieve predesigned migration patterns. ${ }^{28,65,66}$ Fuel density is critical to DNA walkers as the valence of a particle is limited by fuel and DNA densities. The drawback of origami tracks is that their sizes are restricted to hundreds of nanometers, while glass or $\mathrm{Au}$ surfaces can be centimeters. Surface geometry may also impact the migration performances such as walkers on particles ${ }^{40}$, which may behave differently compared to those on flat surfaces.

Effects of cargoes. SV and PS particles of similar sizes do not show significant differences in their migration dynamics. This suggests that migration function relies primarily on the interactions between DNA walker strands and surface RNA fuels, which remain stable with different cargoes. In contrast, the cargo size affects walking motions by changing the number of strands per particle. The 20 -nm-diameter particles have fewer walker strands (estimated to be roughly 20 per particle) bound to fuel strands due to smaller surface areas; the 200-nmdiameter particles have significantly more strands (on the order of 1000 per particle; see SI). Thus, the small particles require less time and fewer enzymes to cleave RNA fuels and move walker strands. As a result, they have kinetically faster turnovers and higher velocities.

Walker-fuel strand designs. The anchored fuel design shows a significant effect on tuning migration modes. Footholds can restrict the walkers from high scaling motions and lead to more random diffusions. This may be explained as footholds significantly increase the energy gap for movement due to hydrogen bonds between DNA strands. The lengths of walker and fuel strands (with no anchor) affect both step lengths and velocities. The activation energy for walking should increase with the length of the walker-fuel pair so that longer strands require more time for cleavage and are kinetically slower. Thus, velocities decrease with increasing 
walker length. Interestingly, longest step lengths are observed with intermediate lengths (13 or 18-nt) as they have longer step times due to their stability. The percentages of different migrations are not affected significantly by walker lengths when they all use the free strand design.

Environmental conditions. Enzyme and $\mathrm{Mg}^{2+}$ concentrations can affect step lengths and velocities of DNA walkers. In a low enzyme concentration range $(<1 \mathrm{nM})$, the velocities and step lengths remain roughly the same. With a high enzyme concentration ( $5 \mathrm{nM})$, the velocities increase drastically. A large quantity of enzymes can accelerate the rate of fuel cleavage, resulting in faster velocity and more hopping particles. Accordingly, the particles under high enzyme concentrations show higher percentages in ballistic and Lévy motions. $\mathrm{Mg}^{2+}$ concentrations impact step lengths in two ways. First, a high $\mathrm{Mg}^{2+}$ concentration will lead to stronger binding between DNA and RNA strands, so the walkers are more stable and move for a longer period of time. On the other hand, a large amount of $\mathrm{Mg}^{2+}$ suppresses enzyme activities (especially $>6 \mathrm{mM}$ ), which will slow the walkers. The balance of these two effects results in longer step lengths at moderate $\mathrm{Mg}^{2+}$ concentrations (12 $\left.\mathrm{mM}\right)$.

Relevance to mathematical models. The random walk model provides new insight into migration dynamics. Different designs and experimental conditions affect distributions of walker steps and further modulate the migration patterns of particles. Experimental results are highly in accordance with model predictions. This suggests that DNA walkers may serve as an excellent platform for validating mathematical models of random motions. Traditional studies use animals as samples, where animal movements are tracked and analyzed to compare with models. Typically, such approaches take several days to months for data collection and require expensive devices for tracking. Microscale systems such as DNA walkers have the advantage of obtaining massive datasets with much less experimental time. We expect that DNA nanotechnology could be widely adopted for biophysical studies in future.

Relevance to natural process. Our findings on migration dynamics and design principles are not specific to our DNA walker system, as we show that cargoes do not affect movement patterns. Random walk models may be adopted for other autonomous DNA motors. ${ }^{65,67}$ Nonautonomous walkers may also be analyzed with sufficient numbers of turnovers. ${ }^{68}$ The distinct migration behaviors observed in this study are common in nature - animals and microorganisms show multiple modes of movements. For example, bacteria can switch between swimming and tumbling. These distinct motions are essential for bacterial chemotaxis ${ }^{69}$. They perform swimming motions in search for food and tumbling behaviors once they find food and will feed themselves by staying in the area. Thus, switchable migration modes may potentially be used for understanding biological processes and developing novel nanodevices. For example, DNA walkers for drug delivery ${ }^{30}$ may perform hopping motions for fast search of targeting spots and diffusive walk for drug release at targeted locations. Such programmable behaviors could drastically improve the efficiency of drug delivery and gain advantages over other methods.

Finally, we explored a random walk model to study DNA walker dynamics and tune their 
migration modes through an extensive mechanistic study. We envision that with fast development of DNA design tools, DNA walker behaviors may be tuned with high precision and active control. Such highly programmable DNA motors will have great potential for biosensors, drug delivery, microscale manufacturing, and other fields as biocompatible nanorobots.

\section{EXPERIMENTAL AND COMPUTATIONAL METHODS}

\section{Materials}

All DNA and RNA sequences were custom-synthesized by Integrated DNA Technologies. The sequence information is listed in supplementary table S1. All lipids were purchased from Avanti Polar Lipids. RNAse $\mathrm{H}$ was purchased from New England Biolabs. PS particles and 1-ethyl3-(3-dimethylaminopropyl) carbodiimide (EDC) hydrochloride were purchased from Thermo Fisher Scientific. All other reagents were purchased from Click Chemistry Tools unless noted otherwise.

\section{Sample preparation}

PS particles were chosen in this study, because of precise control of their sizes. Approximately $10 \mu \mathrm{l}$ stock particles with a diameter of 200 or $20 \mathrm{~nm}$ and $10 \mu \mathrm{l} 1 \mathrm{mM}$ DNA solution were added into $80 \mu \mathrm{l}$ MES (2-(N-morpholino) ethanesulfonic acid) buffer for a 15-minute incubation. Then, roughly $5 \mu \mathrm{l} 100 \mathrm{mM}$ EDC was added to the solution, which was adjusted to $\mathrm{pH} 6.5$ followed by sonication. DNA strands form covalent bonds with the carboxyl groups on particle surfaces via click chemistry. After sonication, the mixture solution was left for overnight incubation. A centrifugation was performed at $20,000 \mathrm{~g}$ for 30 minutes to retrieve the modified particles. The precipitated particles were re-dispersed in $200 \mu$ in $1 \times$ phosphate buffered saline (PBS) buffer.

SV were prepared by introducing DNA-lipid conjugates into lipid composition. DNA-lipid was synthesized by click chemistry with azide modified DNA and 1,2-distearoyl-sn-glycero-3phosphoethanolamine- $\mathrm{N}$-[dibenzocyclooctyl(polyethylene glycol)-2000] (ammonium salt). A dehydration-rehydration method was used for vesicle assembly ${ }^{70}$. Lipid components include 1,2-dimyristoyl-sn-glycero-3-phosphoethanolamine (PE), 1,2-dipalmitoyl-sn-glycero-3phosphocholine (PC), and 1,2-dimyristoyl-sn-glycero-3-phosphoethanolamine-N-(lissamine rhodamine $B$ sufonyl) (ammonium salt) (or Liss Rhod PE). The lipid mixture (PE, PC, Liss Rhod PE) dispersed in chloroform was incubated with the synthesized DNA-lipid conjugate in PBS at a molar ratio of 50:50:1:1, respectively. The mixed solution was dried in a vacuum chamber to form lipid bilayers and rehydrated with $1 \mathrm{ml}$ PBS buffer and heated to $90{ }^{\circ} \mathrm{C}$. The mixture was stirred at $500 \mathrm{rpm}$ for an hour, forming vesicles.

\section{Experimental procedure}

A microfluidic imaging channel was assembled using an RNA-fuel-decorated coverslip and a quartz slide. The detailed procedure was reported elsewhere. ${ }^{47}$ The fuel surface was passivated first, and sufficient PBS solution was supplied into the channel to remove unbound passivation reagents. A prepared particle solution was introduced into the imaging channel by a syringe and incubated for 5 minutes for the particles to bind to the surface. Approximately $500 \mu \mathrm{l}$ PBS buffer was flown into the channel to wash away unbound vesicles. Then, $50 \mu \mathrm{l} 1 \times$ 
nuclease buffer (containing $50 \mathrm{mM}$ Tris- $\mathrm{HCl}, 75 \mathrm{mM} \mathrm{KCl}, 3 \mathrm{mM} \mathrm{MgCl}_{2}$, and $10 \mathrm{mM}$ dithiothreitol or DTT at pH 8.3) was supplied to the channel, followed by introduction of RNase $\mathrm{H}$ (New England Biolabs) solution at a final concentration of $1 \mathrm{nM}$. The particles were then monitored in time-lapse imaging.

\section{MSD scaling calculation derived from CTRW model}

In the standard CTRW model ${ }^{71}$, the density profile has the self-similar form $n(x, t)=$ $(1 / t)^{\theta} \Phi\left(x / t^{\theta}\right)$. The step lengths of the particles are calculated from step time and velocity distributions: 61

$$
g(x)=\int_{-\infty}^{+\infty} d v \int_{0}^{+\infty} \delta(x-v \tau) h(v) F(\tau) d \tau
$$

where $\delta(x-v \tau)$ is the Dirac delta function. $h(v)$ and $F(\tau)$ are the distribution of velocities and step times, as described above. The delta function takes the value of unity when the particle moves for time $\tau$ at a velocity of $v$, which means that the particle has a step length of $x$ for all combinations of velocities and step times. The lengths of particle jumps are modelled with a power law distribution $g(x) \propto x^{-1-2 \beta}$. The exponent $\theta$ in the scaling function $\Phi$ depends on $\beta$ and $\gamma$ in the following way ${ }^{71}$ :

$$
\theta=\left\{\begin{array}{cc}
\gamma / 2 \beta, & 0<\beta<1, \quad 0<\gamma<1 \\
1 / 2 \beta, & 0<\beta<1, \quad \gamma>1 \\
\gamma / 2, \quad \beta>1, \quad 0<\gamma<1
\end{array}\right.
$$

The scaling of different transport regimes is determined by the function $\Phi$. It shows how the spatial expansion of the cloud of random walkers scales with time: $x \propto t^{\theta}$ and in terms of MSD: $\left\langle x^{2}\right\rangle \propto t^{\alpha}$, where $\alpha=2 \theta$.

\section{ACKNOWLEDGEMENT}

This work was supported by the Office of Naval Research. J.H.C. acknowledges support in part from the National Science Foundation. 


\section{REFERENCES}

1. Seeman, N. C., DNA nanotechnology: novel DNA constructions. Annual review of biophysics and biomolecular structure 1998, 27, 225-248.

2. Seeman, N. C., DNA engineering and its application to nanotechnology. Trend in Biotechnology 1999, 17, 437-443.

3. Pinheiro, A. V.; Han, D.; Shih, W. M.; Yan, H., Challenges and opportunities for structural DNA nanotechnology. Nature Nanotechnology 2011, 6, 763-772.

4. Ke, Y.; Castro, C.; Choi, J. H., Structural DNA nanotechnology: artificial nanostructures for biomedical research. Annual review of biomedical engineering 2018, 20, 375-401.

5. Rothemund, P. W., Folding DNA to create nanoscale shapes and patterns. Nature 2006, 440, 297-302.

6. Tian, C.; Li, X.; Li, Z.; Jiang, W.; Wang, G.; Mao, C., Directed Self-Assembly of DNA Tiles into Complex Nanocages. Angewandte Chemie 2014, 126, 8179-8182.

7. Dietz, H.; Douglas, S. M.; Shih, W. M., Folding DNA into Twisted and Curved Nanoscale Shapes. Science 2009, 325, 725-730.

8. Han, D.; Pal, S.; Nangreave, J.; Deng, Z.; Liu, Y.; Yan, H., DNA origami with complex curvatures in three-dimensional space. Science 2011, 332, 342-346.

9. Ke, Y.; Ong, L. L.; Shih, W. M.; Yin, P., Three-dimensional Structures Self-assembled from DNA Bricks. Science 2012, 388, 1177-1183.

10. Yurke, B.; Turberfield, A. J.; Mills Jr, A. P.; Simmel, F. C.; Neumann, J. L., A DNAfuelled molecular machine made of DNA. Nature 2000, 406, 605-608.

11. Simmel, F. C.; Yurke, B., A DNA-based molecular device switchable between three distinct mechanical states. Applied Physics Letters 2002, 80, 883-885.

12. Penchovsky, R.; Breaker, R. R., Computational design and experimental validation of oligonucleotide-sensing allosteric ribozymes. Nature biotechnology 2005, 23, 1424-1433.

13. Seelig, G.; Soloveichik, D.; Zhang, D. Y.; Winfree, E., Enzyme-Free Nucleic Acid Logic Circuits. Science 2006, 314, 1585-1588.

14. Stojanovic, M. N.; Semova, S.; Kolpashchikov, D.; Macdonald, J.; Morgan, C.; Stefanovic, D., Deoxyribozyme-based ligase logic gates and their initial circuits. Journal of the American Chemical Society 2005, 127, 6914-6915.

15. Dannenberg, F.; Kwiatkowska, M.; Thachuk, C.; Turberfield, A. J. In DNA walker circuits: Computational potential, design, and verification, International Workshop on DNABased Computers, Springer: 2013; pp 31-45.

16. Feng, L.; Park, S. H.; Reif, J. H.; Yan, H., A two-state DNA lattice switched by DNA nanoactuator. Angewandte Chemie International Edition 2003, 42, 4342-4346.

17. Yan, H.; Zhang, X.; Shen, Z.; Seeman, N. C., A robust DNA mechanical device controlled by hybridization topology. Nature 2002, 415, 62-65.

18. Shin, J.-S.; Pierce, N. A., A synthetic DNA walker for molecular transport. Journal of the American Chemical Society 2004, 126, 10834-10835.

19. Sherman, W. B.; Seeman, N. C., A precisely controlled DNA biped walking device. Nano letters 2004, 4, 1203-1207.

20. Wang, Z.; Hou, R.; Loh, I. Y., Track-walking molecular motors: a new generation beyond bridge-burning designs. Nanoscale 2019, 11, 9240-9263.

21. Bath, J.; Green, S. J.; Allen, K. E.; Turberfield, A. J., Mechanism for a directional, 
processive, and reversible DNA motor. Small 2009, 5, 1513-1516.

22. Muscat, R. A.; Bath, J.; Turberfield, A. J., A programmable molecular robot. Nano letters 2011, 11, 982-987.

23. Green, S.; Bath, J.; Turberfield, A., Coordinated chemomechanical cycles: a mechanism for autonomous molecular motion. Physical review letters 2008, 101, 238101.

24. Yin, P.; Yan, H.; Daniell, X. G.; Turberfield, A. J.; Reif, J. H., A unidirectional DNA walker that moves autonomously along a track. Angewandte Chemie International Edition 2004, 43, 4906-4911.

25. Tian, Y.; He, Y.; Chen, Y.; Yin, P.; Mao, C., A DNAzyme That Walks Processively and Autonomously along a One-Dimensional Track. Angewandte Chemie International Edition 2005, 44, 4355-4358.

26. Venkataraman, S.; Dirks, R. M.; Rothemund, P. W.; Winfree, E.; Pierce, N. A., An autonomous polymerization motor powered by DNA hybridization. Nature Nanotechnology 2007, 2, 490-494.

27. You, M.; Chen, Y.; Zhang, X.; Liu, H.; Wang, R.; Wang, K.; Williams, K. R.; Tan, W., An Autonomous and Controllable Light-Driven DNA Walking Device. Angewandte Chemie International Edition 2012, 51, 2457-2460.

28. Gu, H.; Chao, J.; Xiao, S. J.; Seeman, N. C., A Proximity-based Programmable DNA Nanoscale Assembly Line. Nature 2010, 465, 202-205.

29. Thubagere, A. J.; Li, W.; Johnson, R. F.; Chen, Z.; Doroudi, S.; Lee, Y. L.; Izatt, G.; Wittman, S.; Srinivas, N.; Woods, D., A cargo-sorting DNA robot. Science 2017, 357, eaan6558.

30. Li, F.; Cha, T. G.; Pan, J.; Ozcelikkale, A.; Han, B.; Choi, J. H., DNA WalkerRegulated Cancer Cell Growth Inhibition. ChemBioChem 2016, 17, 1138-1141.

31. McKee, M. L.; Milnes, P. J.; Bath, J.; Stulz, E.; O’Reilly, R. K.; Turberfield, A. J., Programmable one-pot multistep organic synthesis using DNA junctions. Journal of the American Chemical Society 2012, 134, 1446-1449.

32. He, Y.; Liu, D. R., Autonomous multistep organic synthesis in a single isothermal solution mediated by a DNA walker. Nature nanotechnology 2010, 5, 778-782.

33. Wang, Z.-G.; Elbaz, J.; Willner, I., DNA machines: bipedal walker and stepper. Nano letters 2011, 11, 304-309.

34. Pan, J.; Li, F.; Cha, T.-G.; Chen, H.; Choi, J. H., Recent progress on DNA based walkers. Current opinion in biotechnology 2015, 34, 56-64.

35. Li, J.; Johnson-Buck, A.; Yang, Y. R.; Shih, W. M.; Yan, H.; Walter, N. G., Exploring the speed limit of toehold exchange with a cartwheeling DNA acrobat. Nature nanotechnology 2018, 13, 723-729.

36. Bazrafshan, A.; Meyer, T.; Su, H.; Brockman, J. M.; Blanchard, A. T.; Piranej, S.; Duan, Y.; Ke, Y.; Salaita, K., Tunable DNA Origami Motors Translocate Ballistically Over $\mu \mathrm{m}$ Distances at $\mathrm{nm} / \mathrm{s}$ Speeds. Angewandte Chemie International Edition 2020.

37. Wang, Z.; Hou, R.; Efremov, A., Directional fidelity of nanoscale motors and particles is limited by the 2nd law of thermodynamics-Via a universal equality. The Journal of chemical physics 2013, 139, 035105.

38. Samii, L.; Blab, G. A.; Bromley, E. H.; Linke, H.; Curmi, P. M.; Zuckermann, M. J.; Forde, N. R., Time-dependent motor properties of multipedal molecular spiders. Physical 
Review E 2011, 84, 031111.

39. Khara, D. C.; Schreck, J. S.; Tomov, T. E.; Berger, Y.; Ouldridge, T. E.; Doye, J. P.; Nir, E., DNA bipedal motor walking dynamics: an experimental and theoretical study of the dependency on step size. Nucleic acids research 2018, 46, 1553-1561.

40. Jung, C.; Allen, P.; Ellington, A., A stochastic DNA walker that traverses a microparticle surface. Nature nanotechnology 2016, 11, 157-163.

41. Cha, T.-G.; Pan, J.; Chen, H.; Robinson, H. N.; Li, X.; Mao, C.; Choi, J. H., Design principles of DNA enzyme-based walkers: Translocation kinetics and photoregulation. Journal of the American Chemical Society 2015, 137, 9429-9437.

42. Semenov, O.; Olah, M. J.; Stefanovic, D., Cooperative linear cargo transport with molecular spiders. Natural Computing 2013, 12, 259-276.

43. Olah, M. J.; Stefanovic, D., Superdiffusive transport by multivalent molecular walkers moving under load. Physical Review E 2013, 87, 062713.

44. Ouldridge, T. E.; Hoare, R. L.; Louis, A. A.; Doye, J. P.; Bath, J.; Turberfield, A. J., Optimizing DNA nanotechnology through coarse-grained modeling: a two-footed DNA walker. ACS nano 2013, 7, 2479-2490.

45. Zuckermann, M. J.; Angstmann, C. N.; Schmitt, R.; Blab, G. A.; Bromley, E. H.; Forde, N. R.; Linke, H.; Curmi, P. M., Motor properties from persistence: a linear molecular walker lacking spatial and temporal asymmetry. New Journal of Physics 2015, 17, 055017.

46. Simmel, F. C., Processive motion of bipedal DNA walkers. ChemPhysChem 2009, 10, 2593-2597.

47. Pan, J.; Du, Y.; Qui, H.; Upton, L. R.; Li, F.; Choi, J. H., Mimicking Chemotactic Cell Migration with DNA Programmable Synthetic Vesicles. Nano letters 2019, 19, 9138-9144.

48. Yehl, K.; Mugler, A.; Vivek, S.; Liu, Y.; Zhang, Y.; Fan, M.; Weeks, E. R.; Salaita, K., High-speed DNA-based rolling motors powered by RNase H. Nature nanotechnology 2016, 11, 184-190.

49. Kenkre, V.; Montroll, E.; Shlesinger, M., Generalized master equations for continuoustime random walks. Journal of Statistical Physics 1973, 9, 45-50.

50. Klages, R.; Radons, G.; Sokolov, I. M., Anomalous transport: foundations and applications. John Wiley \& Sons: 2008.

51. Bath, J.; Green, S. J.; Turberfield, A. J., A Free-Running DNA Motor Powered by a Nicking Enzyme. Angewandte Chemie 2005, 44, 4358-4361.

52. Cha, T.-G.; Pan, J.; Chen, H.; Salgado, J.; Li, X.; Mao, C.; Choi, J. H., A synthetic DNA motor that transports nanoparticles along carbon nanotubes. Nature nanotechnology 2014, 9, 39-43.

53. Qu, X.; Zhu, D.; Yao, G.; Su, S.; Chao, J.; Liu, H.; Zuo, X.; Wang, L.; Shi, J.; Wang, L., An exonuclease III-powered, on-particle stochastic DNA walker. Angewandte Chemie International Edition 2017, 56, 1855-1858.

54. Michalet, X., Mean square displacement analysis of single-particle trajectories with localization error: Brownian motion in an isotropic medium. Physical Review E 2010, 82, 041914.

55. Amit, D. J.; Parisi, G.; Peliti, L., Asymptotic behavior of the" true" self-avoiding walk. Physical Review B 1983, 27, 1635.

56. Andreanov, A.; Grebenkov, D. S., Time-averaged MSD of Brownian motion. Journal of 
Statistical Mechanics: Theory and Experiment 2012, P07001.

57. Semenov, O.; Olah, M. J.; Stefanovic, D., Mechanism of diffusive transport in molecular spider models. Physical Review E 2011, 83, 021117.

58. Rhee, I.; Shin, M.; Hong, S.; Lee, K.; Kim, S. J.; Chong, S., On the levy-walk nature of human mobility. IEEE/ACM transactions on networking 2011, 19, 630-643.

59. Raichlen, D. A.; Wood, B. M.; Gordon, A. D.; Mabulla, A. Z.; Marlowe, F. W.; Pontzer, $\mathrm{H}$., Evidence of Lévy walk foraging patterns in human hunter-gatherers. Proceedings of the National Academy of Sciences 2014, 111, 728-733.

60. Metzler, R.; Jeon, J.-H.; Cherstvy, A. G.; Barkai, E., Anomalous diffusion models and their properties: non-stationarity, non-ergodicity, and ageing at the centenary of single particle tracking. Physical Chemistry Chemical Physics 2014, 16, 24128-24164.

61. Zaburdaev, V.; Schmiedeberg, M.; Stark, H., Random walks with random velocities. Physical Review E 2008, 78, 011119.

62. Klafter, J.; Blumen, A.; Shlesinger, M. F., Stochastic pathway to anomalous diffusion. Physical Review A 1987, 35, 3081.

63. Alstott, J.; Bullmore, D. P., powerlaw: a Python package for analysis of heavy-tailed distributions. PloS one 2014, 9, e85777.

64. Elani, Y.; Law, R. V.; Ces, O., Vesicle-based artificial cells as chemical microreactors with spatially segregated reaction pathways. Nature communications 2014, 5, 5305.

65. Lund, K.; Manzo, A.; Dabby, N.; Michelotti, N.; Johnson-Buck, A.; Nangreave, J.; Taylor, S.; Pei, R.; Stojanovic, M. N.; Walter, N. G.; Winfree, E.; Yan, H., Molecular Robots Guided by Prescriptive Landscapes. Nature 2010, 465, 206-210.

66. Wickham, S. F. J.; Endo, M.; Katsuda, Y.; Hidaka, K.; Bath, J.; Sugiyama, H.; Turberfield, A. J., Direct Observation of Stepwise Movement of a Synthetic Molecular Transporter. Nature Nanotechnology 2011, 6, 166-169.

67. Pan, J.; Cha, T.-G.; Li, F.; Chen, H.; Bragg, N. A.; Choi, J. H., Visible/near-infrared subdiffraction imaging reveals the stochastic nature of DNA walkers. Science advances 2017, 3, e1601600.

68. Tomov, T. E.; Tsukanov, R.; Liber, M.; Masoud, R.; Plavner, N.; Nir, E., Rational design of DNA motors: fuel optimization through single-molecule fluorescence. Journal of the American Chemical Society 2013, 135, 11935-11941.

69. Sidortsov, M.; Morgenstern, Y.; Be'er, A., Role of tumbling in bacterial swarming. Physical Review E 2017, 96, 022407.

70. Kirby, C.; Gregoriadis, G., Dehydration-rehydration vesicles: a simple method for high yield drug entrapment in liposomes. Bio/Technology 1984, 2, 979-984.

71. Metzler, R.; Klafter, J., The random walk's guide to anomalous diffusion: a fractional dynamics approach. Physics reports 2000, 339, 1-77. 


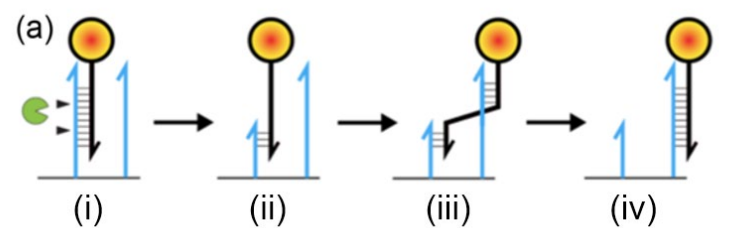

(b)

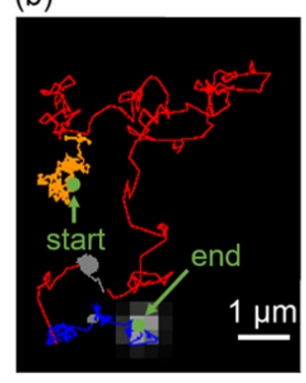

(c)

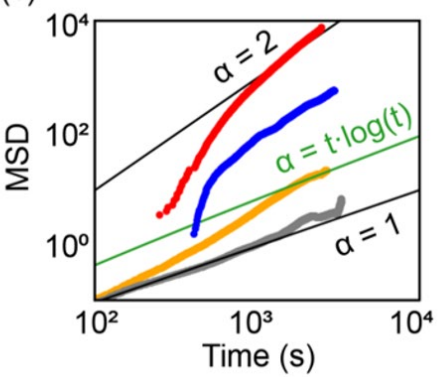

Figure 1. Classification of migration modes of cargo-moving DNA walkers. (a) Scheme of enzymatic burnt-bridge mechanism. Blue and black lines represent RNA fuels and DNA walker strands, respectively. Orange circles indicate the cargo. The enzyme (shown in green) cleaves RNA in the RNA/DNA hybrid (state (i) $\rightarrow$ (ii)). Then, the DNA strands search and bind with next available fuel (state (iii)). The DNA strand finally moves to the new fuel via branch migration (state (iv)). (b) Trajectory of a single particle exhibiting distinct migration modes. A 20-nmdiameter PS particle is monitored using TIRF microscopy for 2 hours, while migrating on a glass coverslip functionalized with RNA fuels under $1 \mathrm{nM}$ enzyme and $3 \mathrm{mM} \mathrm{Mg}^{2+}$. The start and end locations are marked in green color. The particle started with a self-avoiding walk (orange) and transitioned to a ballistic motion with multiple long-distance jumps (red). Then, the particle was restricted within a small area, showing diffusive motions (gray). The particle later moved out of the restricted area and transitioned to a Lévy mode (blue). (c) Representative MSD plots of different walking modes. The two thin black lines represent pure diffusion ( $\alpha=1$ ) and ballistic motion ( $\alpha=2$ ), where $\alpha$ denotes the scaling exponent of MSD. The green curve shows the theoretical prediction of self-avoiding walk: $\alpha=t \cdot \log (t)$, where $t$ is time. The gray line is an example of diffusion and matches well with diffusion theory. The orange line shows self-avoiding movement from the experiment. Experimental Lévy flight (blue) falls between ballistic and self-avoiding lines. Distinct deviation from the self-avoiding MSD can be observed. The measured ballistic motion (red) follows the $\alpha=2$ line after 1000 seconds. Note that all the migration modes follow the diffusion in a short time period and differentiate into four distinct modes at a larger timescale. The transition results from the timeaveraged MSD used in the analysis, showing a more accurate estimation for overall MSD in the long term. 

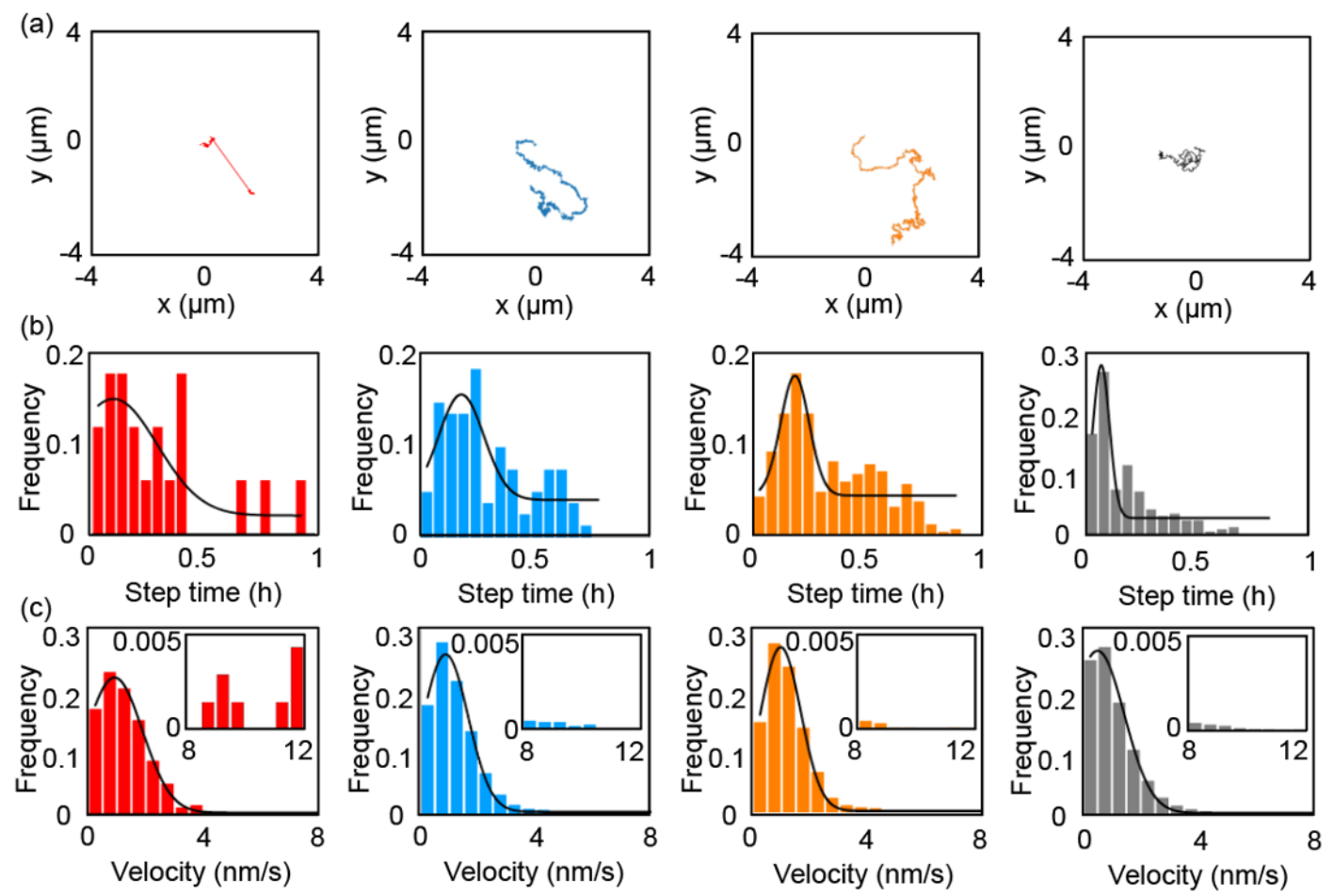

Figure 2. Comparison of four migration modes. (a) Representative trajectory of each mode, starting from $(0,0)$. The ballistic mode (red) exhibits jumpy steps. Lévy type (blue) and selfavoiding motion (orange) show similar trajectories, while their MSD behaviors are distinct and different as seen in Figure 1. Diffusion (gray) demonstrates small steps in a restricted small area. (b) Step time distributions of different walking modes. The histograms are fitted to Gaussian functions to extract statistical features. The red histograms are ballistic motions and show a broad distribution in step time. The blue histograms are Lévy particles whose distribution is broader than that of self-avoiding walkers (orange) although they are narrower compared to the ballistic motion. Diffusion (gray) shows an extremely narrow distribution of step time. (c) Velocity distributions of different modes. In a low velocity range (e.g., $0 \sim 8 \mathrm{~nm} / \mathrm{s}$ ), the distributions are mostly similar. The ballistic mode shows high velocities due to jumps. 


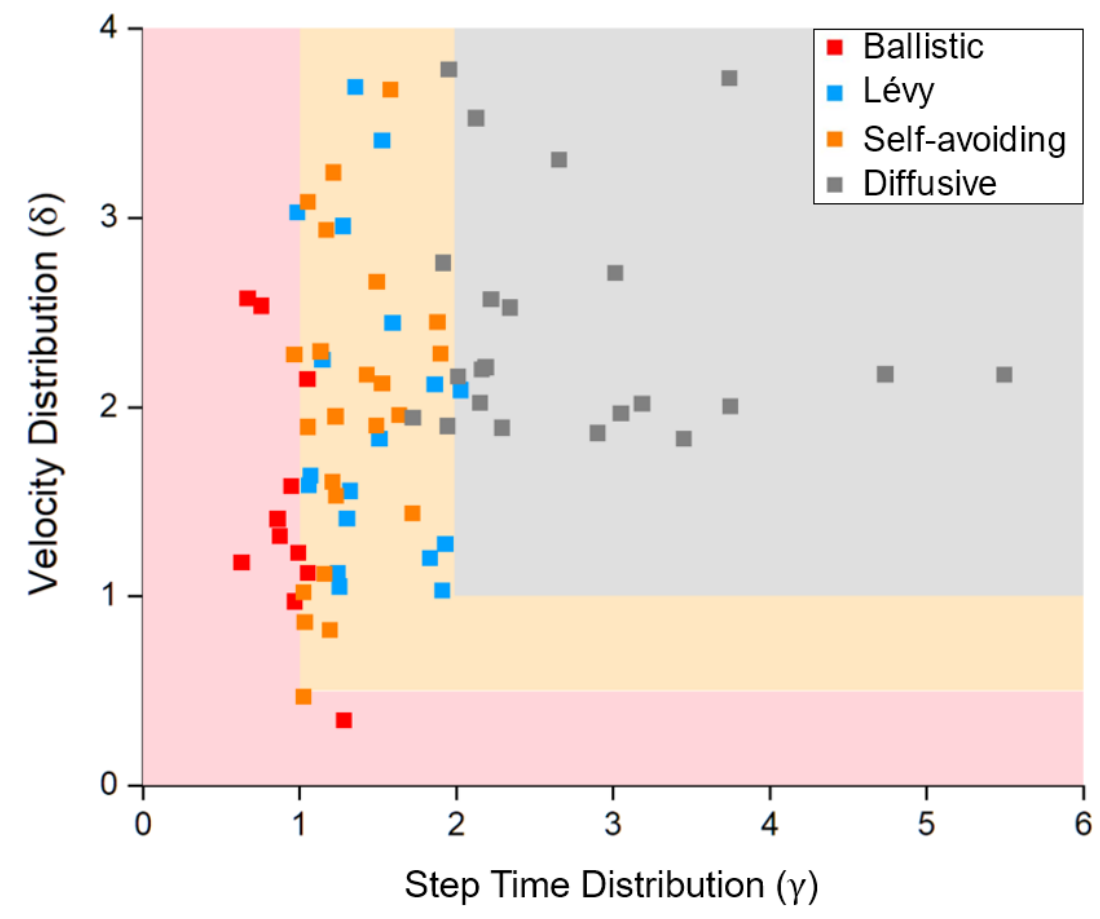

Figure 3. Random walk model with random velocity. The random walk model shows distinct color-coded regimes. Red background shows a theoretical range of ballistic movement. Light brown shade represents super-diffusion including Lévy and self-avoiding motion. Diffusive range is rendered with gray background. Squares denote experimental data and are characterized as different walking modes. It is evident that experimental data marked with color code falls correctly into predicted ranges of step time and velocity distributions. 

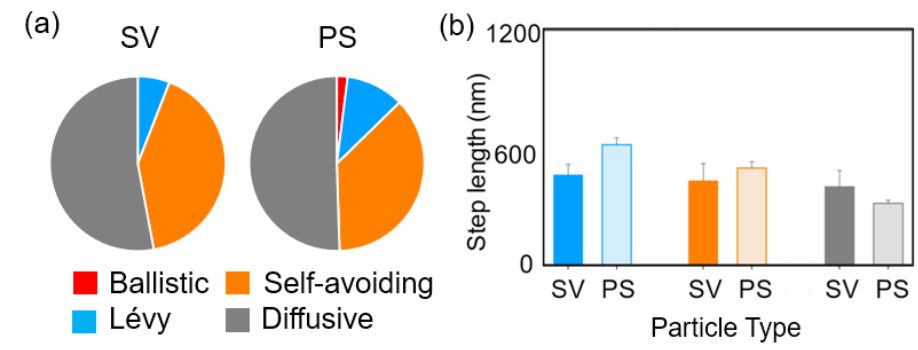

(d)
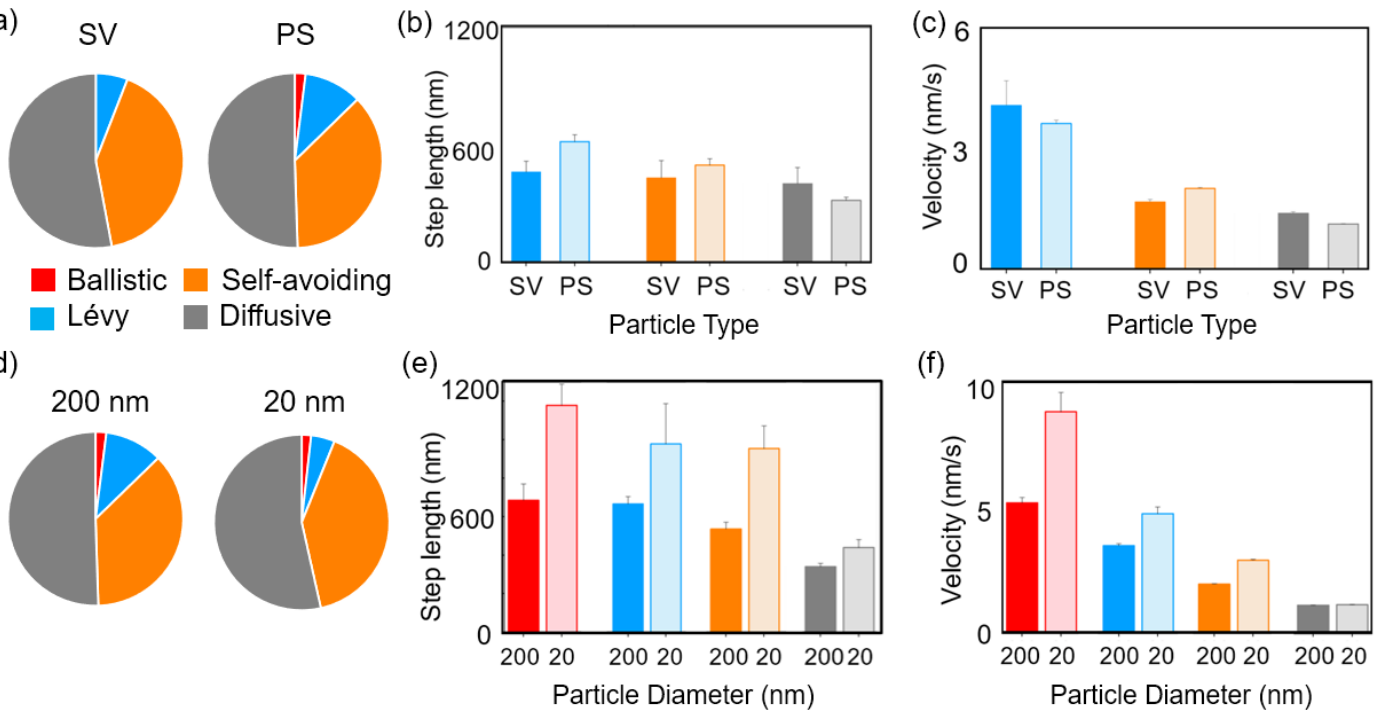

Figure 4. Effects of particle type and size. The experiments were conducted with $1 \mathrm{nM}$ enzyme and $3 \mathrm{mM} \mathrm{Mg}^{2+}$. Ballistic motions are plotted in red. Lévy movements are shown in blue. Selfavoiding and diffusive walks are represented in orange and gray, respectively. (a) Pie charts (i.e., percentages) of different migration modes with SV and PS particles. These two particle types show no significant differences in mode percentages. (b) Average step lengths of the particles. The average step lengths of SV are shown in solid histograms, while those of PS particles are plotted with opaque histograms. Both particles have mostly similar average step lengths regardless of walking modes. (c) Average velocities of SV and PS particles. Their average velocities are similar in all modes. (d) Percentages of different modes for PS particles with a diameter of 200 and $20 \mathrm{~nm}$. No significance differences are observed in mode percentages. (e) Average step lengths of 200 and $20 \mathrm{~nm}$ particles. $20 \mathrm{~nm}$ particles show significantly greater step lengths in all modes. (f) Average velocities of 200 and $20 \mathrm{~nm}$ particles. The average velocities of $20 \mathrm{~nm}$ particles are higher than those of $200 \mathrm{~nm}$ particles. 
(a)

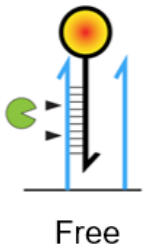

(b)

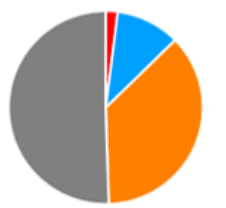

Ballistic Lévy

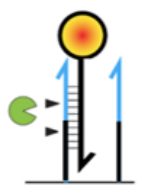

Anchored

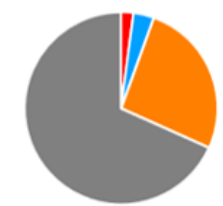

Self-avoiding

Diffusive (c)

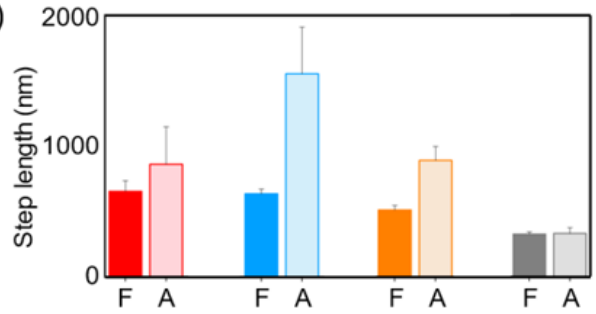

Fuel Design

(d)

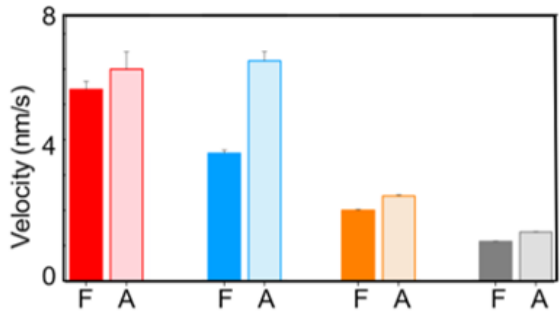

Fuel Design

Figure 5. Effects of foothold designs. The 200-nm-diameter PS particles were used for the experiments under concentrations of $3 \mathrm{mM} \mathrm{Mg}^{2+}$ and $1 \mathrm{nM}$ enzyme. (a) Schematic of free and anchored strand design. In the free design, the fuel strand consists of all RNA, thus it will be fully cleaved by the enzyme. In the anchored design, the lower part (7-nt) of the fuel is replaced by DNA (shown in black), which will remain intact after cleavage. (b) Pie charts (percentages) of migration modes for free (left) and anchored (right) strand designs. The particles with free fuel strands show more self-avoiding and Lévy motions, while diffusion is predominant with anchored strands. (c) Average step lengths for two fuel designs. The average step lengths of free strands (F) are plotted in solid histograms, while those of anchored strands (A) are represented by opaque histograms. Generally, the particles show longer step lengths with anchored strands. (d) Average velocities depending on the fuel design. The velocities with anchored strands are generally higher than those with free strands. 

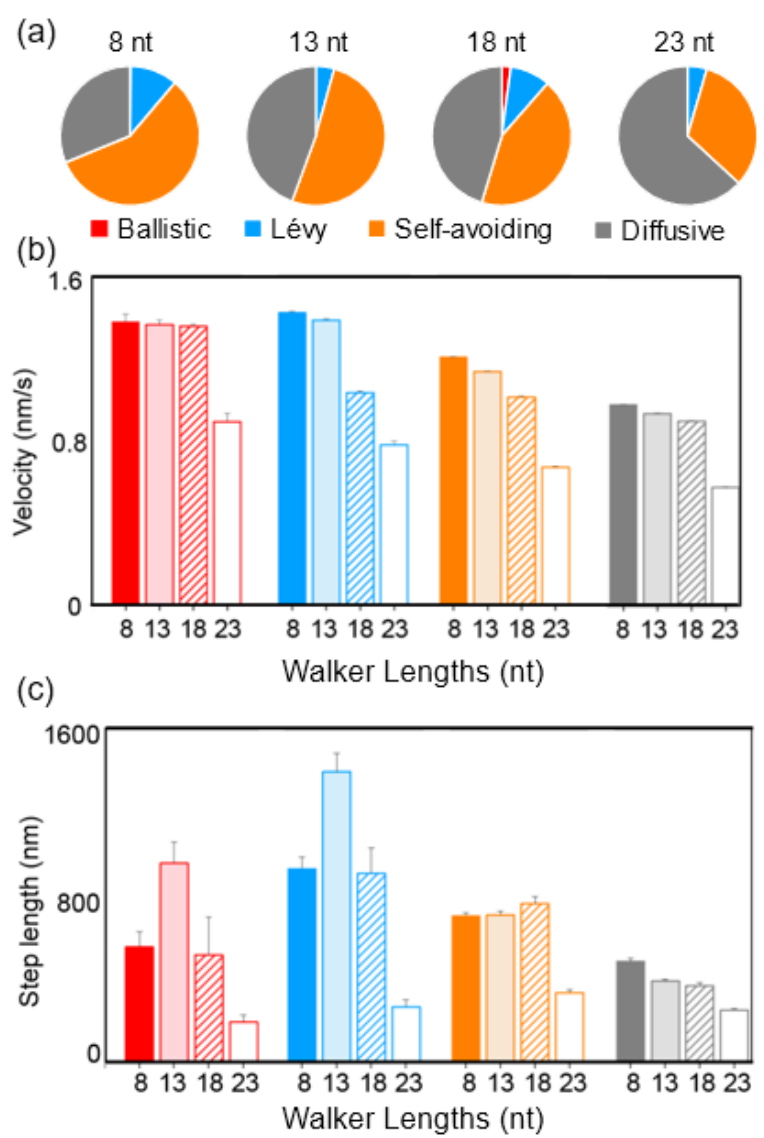

Figure 6. Effects of walker/fuel lengths. Experiments were performed with SV particles under $12 \mathrm{mM} \mathrm{Mg}^{2+}$ and $1 \mathrm{nM}$ enzyme. (a) Pie charts (percentages) of migration modes for a DNA length of $8,13,18$, and 23-nt. Longer DNA shows more diffusive and less self-avoiding behaviors. (b) Average velocities. The average velocities of 8-nt strands are represented by solid histograms, while those of 13-nt are shown with opaque histograms. The results with 18nt strands are given by columns of dashed lines, and those with 23-nt strands are plotted without filling. Longer strands demonstrate reduced velocities in all modes. (c) Average step lengths of different strand lengths. Walkers of 13 or 18-nt lengths show longer step lengths than 8 or 23-nt DNA. 
(a)
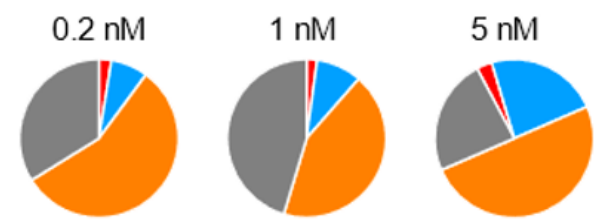

(b)

- Ballistic = Lévy $=$ Self-avoiding $\mathbf{n}$ Diffusive

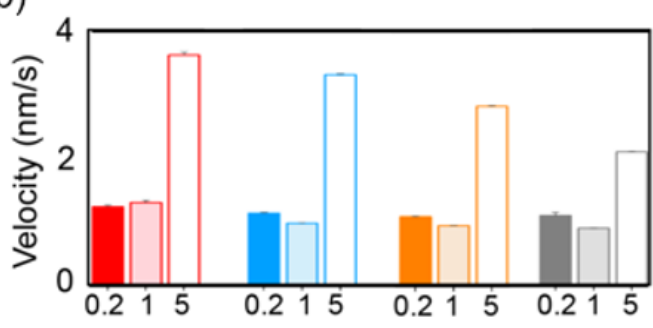

(c)

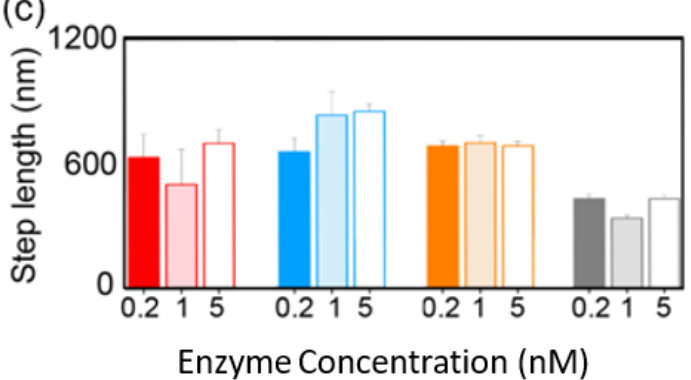

(d)
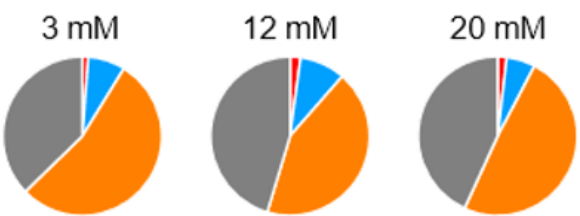

(e)

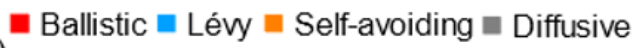

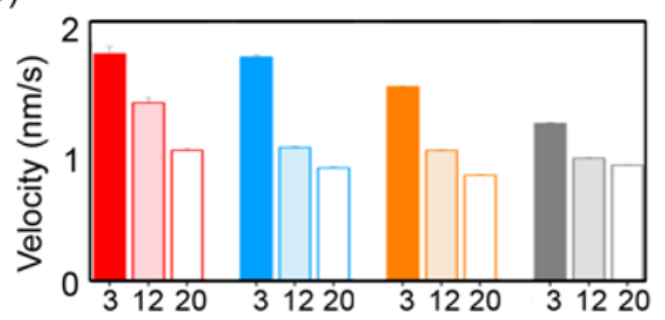

(f)

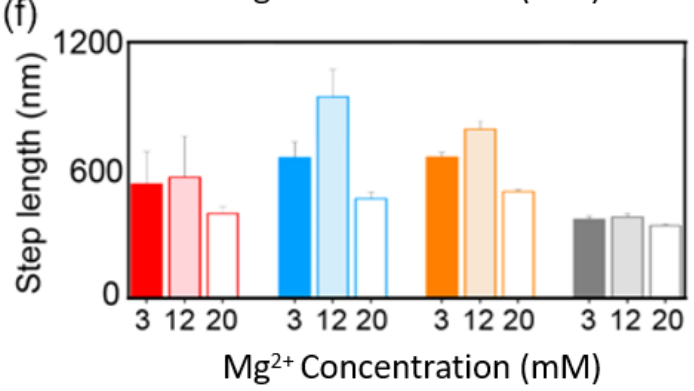

Figure 7. Effects of environmental conditions. The SV particles with 18-nt walker DNA were used in migration experiments. (a) Pie charts (percentages) of four migration modes depending on the enzyme concentration. The ballistic and Lévy walkers are significantly increased at high enzyme concentration. (b) Average velocities of particles at $0.2,1$, and $5 \mathrm{nM}$ enzymes. With a large quantity of enzymes $(5 \mathrm{nM})$, the walkers demonstrate much higher velocities. (c) Average step lengths for different enzyme concentrations. The step lengths do not show significant dependence of enzyme concentration. (d) Percentages of migration modes with different $\mathrm{Mg}^{2+}$ concentrations. No significant changes are observed with different $\mathrm{Mg}^{2+}$ concentrations. (e) Average velocities at 3, 12, and $20 \mathrm{mM} \mathrm{Mg}^{2+}$. The velocities decrease with increasing $\mathrm{Mg}^{2+}$ concentrations. (f) Average step lengths with various amounts of $\mathrm{Mg}^{2+}$. Longest step lengths are observed with $12 \mathrm{mM} \mathrm{Mg}^{2+}$. 